\title{
USO DE COLETORES SOLARES NO ENSINO, EXTENSÃO E EMPREENDEDORISMO
}

DOI: 10.37702/2175-957X.COBENGE.2021.3434

Luiz Carlos Cordeiro Jr - luiz.cordeiro@fat.uerj.br

UERJ

Rua Benedito Alves de Moraes 157

27523-116 - Resende - RJ

Amyr Saraiva Marassi - amyr.saraiva.marassi@hotmail.com

Universidade do Estado do Rio de Janeiro

Rua Professora Dona Betina 408

27570-000 - Porto Real - RJ

Rodrigo de Souza motta Azevedo - rodrigodsmotta@gmail.com

UERJ Fat

Rua valdemir Almeida viana 99

27542-050 - Resende - RJ

Resumo: No artigo proposto, é apresentado o desenvolvimento teórico-experimental de uma bancada didática para análise de eficiência térmica de um coletor solar de placa plana, em complemento a formação da disciplina de Fenômenos de Transporte Experimental do curso de Engenharia Mecânica da Universidade do Estado do Rio de Janeiro, unidade Resende. Inicialmente os alunos são estimulados a desenvolver o modelo analítico do coletor solar, conciliando toda a formação acadêmica nas disciplinas de transferência de calor e mecânica dos Fluidos. No desenvolvimento do experimento, são inseridas as técnicas de aquisição de dados, por intermédio do uso de diversos meios de medição de temperatura e vazão, assim como consulta a dados oficiais de monitoramento ambiental. Em paralelo, os alunos voluntários são estimulados a participar de atividades extensionistas, "Projeto UERJ no Campo" no qual busca atender as necessidades das comunidades rurais, através deste recurso renovável, desenvolvendo nestes alunos competências e estimulando ações empreendedoras na busca de soluções que possam atender a esta comunidade conciliando custo e benefício. $O$ projeto extensionista se estende também a outras atribuições, como meio de propagação de informação, conhecimento, inclusão e acessibilidade, estimulando os alunos a ministrar palestras e oficinas tanto para a comunidade 


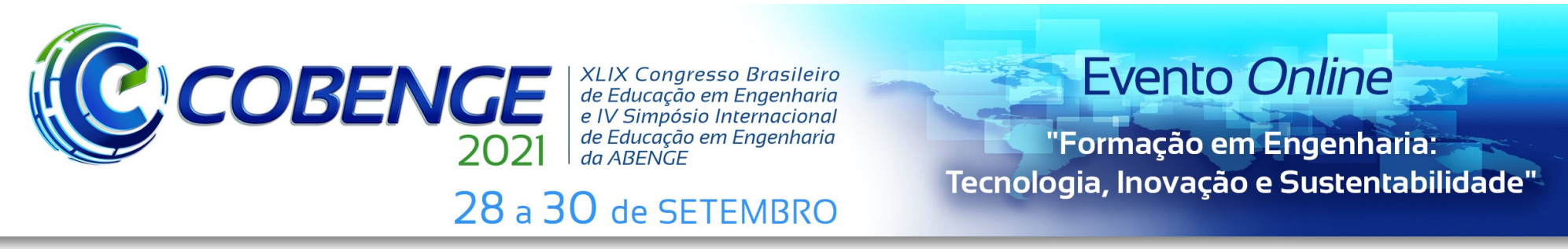

rural como escolas públicas e privadas, da região Sul-Fluminense.

Palavras-chave: Fenômenos de Transporte Experimental; Coletor Solar de Placa Plana; Atividades Extensionistas. 


\section{USO DE COLETORES SOLARES NO ENSINO, EXTENSÃO E EMPREENDEDORISMO}

\section{INTRODUÇÃO}

A sociedade mundial vem gradativamente enfrentando desafios na substituição ou no equilíbrio da matriz energética através do uso de fontes alternativas de energia renovável. Segundo o Atlas Brasileiro de energia solar (2006), o Brasil tem um grande potencial na utilização da energia solar e tem despertado interesse devido ao grande potencial de aproveitamento frente a sua posição geográfica, e estima-se que o aquecimento de água seja responsável por $25 \%$ do consumo energético residencial do país há mais de uma década. Com base nessa afirmativa, a proposta de trabalho a ser desenvolvida pelo Laboratório de Fenômenos de Transporte é de incentivar os alunos na análise e no desenvolvimento do conceito de utilização de um coletor solar, através de uma proposta de "Trilha do Conhecimento". A UERJ-Resende dispõe atualmente de uma área de testes conforme mostrado na figura 1 e figura 2, com diversos modelos conceituais de coletores e que contribuem na formação acadêmica do corpo discente, frente as análises de performance térmica comparativa de cada modelo. O coletor solar escolhido para a realização das atividades foi o de placa plana (CPP) (figura 3), devido sua robustez, simplicidade, baixa manutenção e são consideravelmente menos custosos que outros modelos de coletores no mercado brasileiro atual e são utilizados principalmente para o aquecimento da água, aquecimento de residências, ar condicionado e alguns processos de aquecimento industrial.

Figura 1: Área externa de testes.

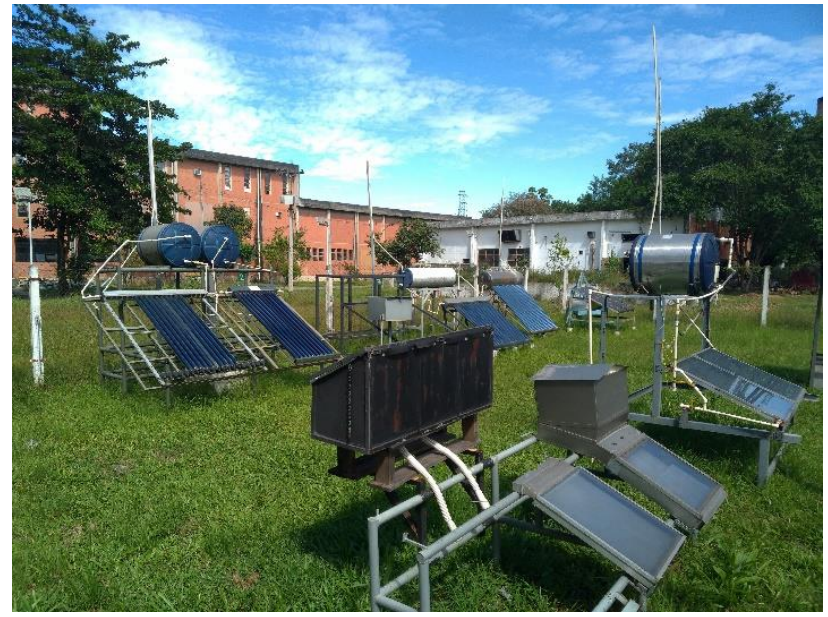

Fonte: Próprio Autor
Figura 2: Vista lateral da área externa de testes.

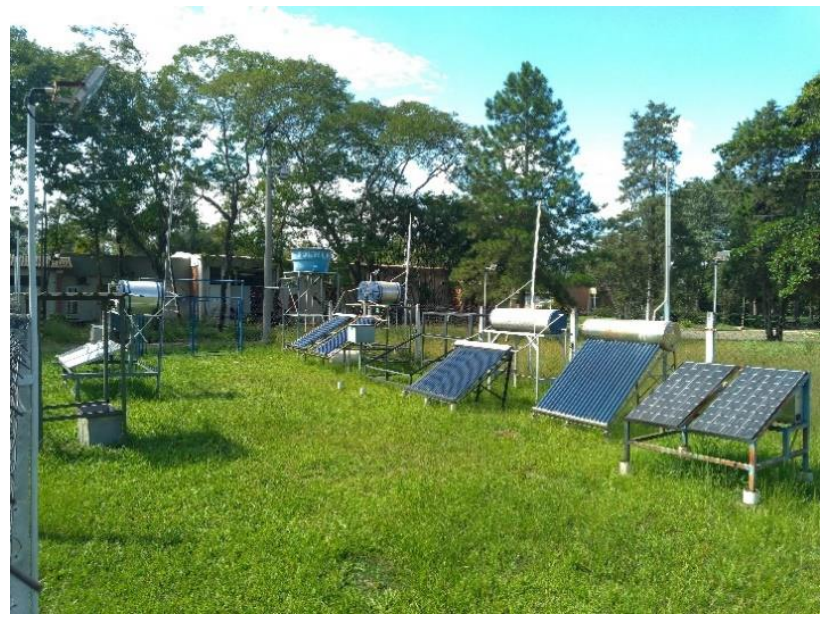

Fonte: Próprio Autor

Figura 3: Modelo de coletor de placa plana escolhido. 


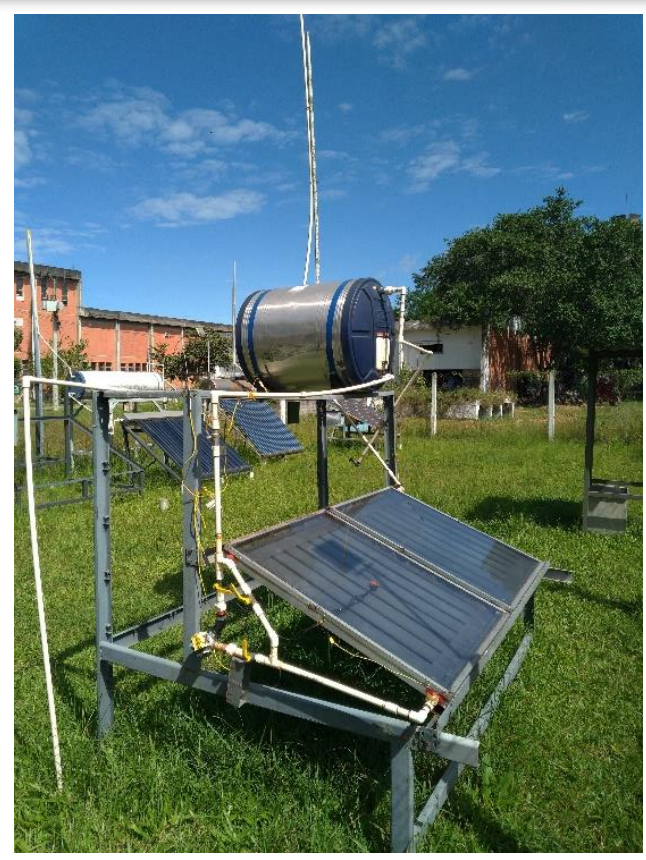

Fonte: Próprio Autor

A análise do coletor de placa plana (CPP) contribuirá no ensino dos alunos do curso de engenharia, onde aprenderão a fazer o dimensionamento correto de um coletor, através da implementação do modelo analítico que utilize as ferramentas de transferência de calor e mecânica dos fluidos, buscando como resultado a aproximação de dados teóricoexperimental.

Para otimizar a proposta de trabalho, foi desenvolvido um modelo pedagógico que concilia a entrega de uma "Trilha de Conhecimento" e em paralelo fomenta nos alunos a necessidade de solução do problema através de metodologia ativa, fazendo com que os alunos trabalhem as competências ou habilidades especificas para análise do modelo.

Por fim, os alunos são convidados a participar de atividades extensionistas, e de forma voluntária contribuir no projeto de Extensão UERJ no Campo, na divulgação dos benefícios de utilização do coletor solar como suporte ao processo produtivo nas propriedades rurais, esterilização de equipamentos e limpeza de ambientes, assim como estender benefícios de conforto aos familiares para utilização pessoal. Esta atividade é possível em virtude do desenvolvimento de um Coletor Solar de Placa Plana, no qual o mesmo foi construído em parceria da UERJ e do Sindicato Rural de Resende, denominado "Coletor Itinerante", o mesmo foi desenvolvido para demonstração nas propriedades rurais por um prazo pré-definido entre sindicato e o produtor. Toda a estrutura é desmontável, e os próprios alunos podem acompanhar a entrega do equipamento, realização da instalação e a apresentação do recurso e benefício do uso das energias renováveis. Os alunos também são estimulados a desenvolver oficinas e palestras à comunidade rural, sendo atualmente desenvolvido através de atividades virtuais, auxiliando a sanar dúvidas sobre a utilização desse equipamento e seus benefícios através da energia solar. Esse complemento de atividade extensionista é um diferencial para formação acadêmica do corpo discente, conciliando todos os pilares necessários para estruturação da casa da excelência (figura 4), buscado entender as necessidades e melhorias, fazendo com que adquirem maturidade, reponsabilidade e profissionalismo.

Figura 4: Casa da excelência. 


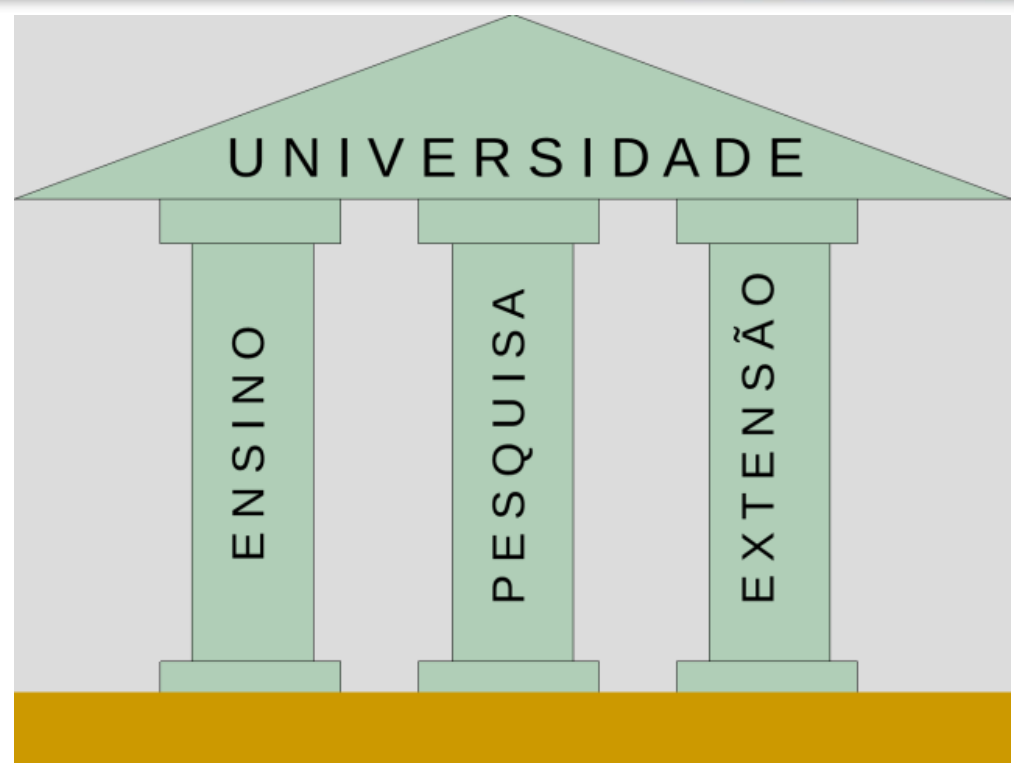

Fonte: Próprio Autor

\section{METODOLOGIA}

Este trabalho foi conduzido no Laboratório de Fenômenos de Transporte Experimental em parceria com a Área de testes do Centro de Fontes Renováveis, ambos localizados na UERJ Campus Resende. No experimento foram utilizados, um (1) reservatório de água de 200 litros (boiler), dois (2) coletores solares de placa plana, registros e tubulações adequadas a altas temperaturas, conforme figura 3. Todo o equipamento foi instrumentado, com termopares tipo $\mathrm{K}$, nas posições indicadas na figura 5 , com os seguintes pontos de instrumentação:

- Temperatura ambiente;

- Entrada e saída do conjunto das placas coletoras, com o objetivo de mapear o ganho de temperatura do fluido, na sua passagem pelo trocador;

- Na superfície externa e interna da cobertura de vidro, alinhados simetricamente e na posição central do vidro, para registro da temperatura incidente e absorvida sobre esta superfície;

- Na superfície da placa absorvedora do coletor solar, valor este utilizado para o modelo analítico proposto;

- Um termopar fixado entre a cobertura e a placa absorvedora;

- Na entrada de alimentação do reservatório de água (boiler);

- Na saída do circuito para atender o consumo, onde será realizada a medição da temperatura final da água para processo.

Figura 5: Disposição dos termopares. 


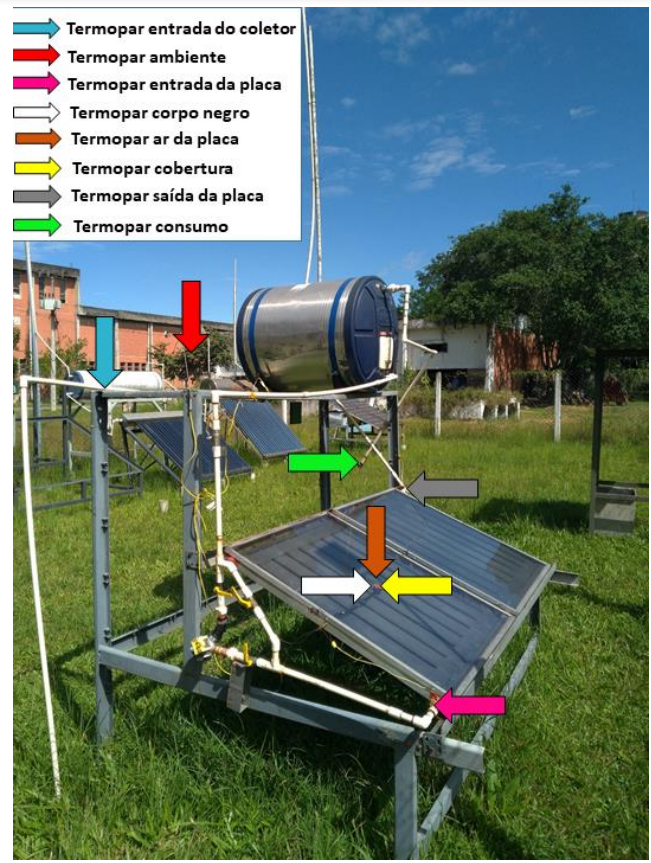

Fonte: Próprio Autor

Para a análise do equipamento em relação a condição de performance energética, e atendendo a condição da conservação de massa, foi imposto ao sistema a inclusão de uma bomba d'água de baixa vazão pilotada por um regulador de tensão (mostrada na figura 6), sendo que a vazão do sistema foi aferida pelo método de galonagem.

Figura 6: Bomba d'água instalada no sistema.

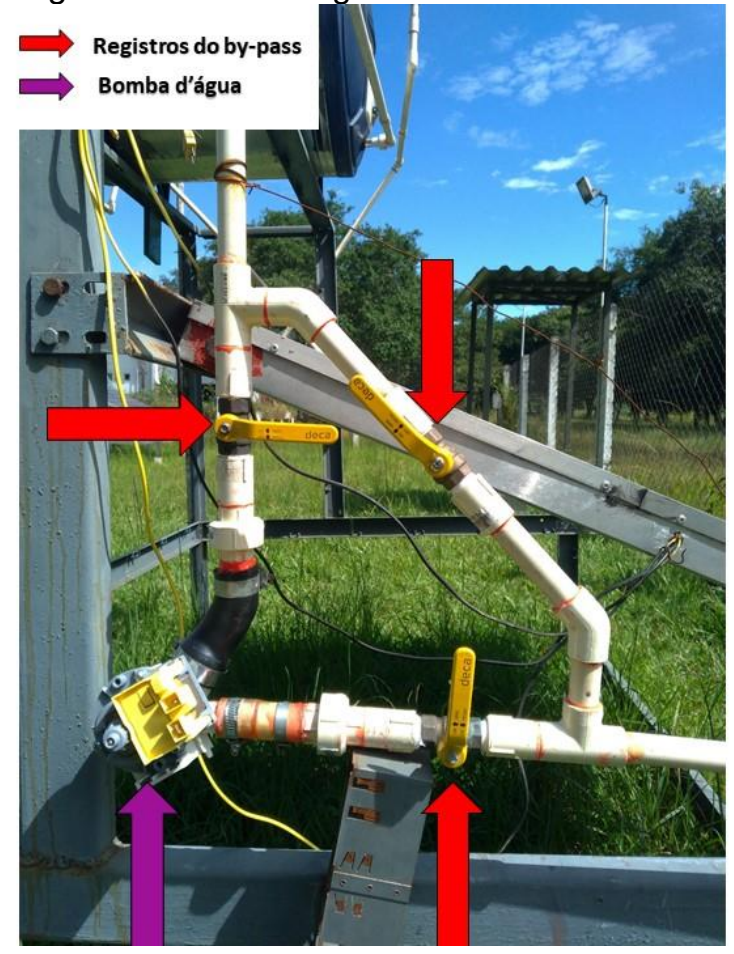

Fonte: Próprio Autor

Para o monitoramento do funcionamento do coletor, os alunos são orientados no correto procedimento da aquisição de dados, assim como identificar variáveis mais complexas através de consulta ao sistema Instituto Nacional de Meteorologia (INMET), conforme será mostrado na "Trilha do Conhecimento". 
As partes importantes da estrutura de um coletor solar para se entender o modelo analítico são: A superfície negra absorvedora de energia solar, que transfere a energia absorvida para o fluido de trabalho, placa transparente acima da superfície absorvedora que reduz a perda por radiação e convecção para o ambiente externo, e um isolamento embaixo e nas bordas da superfície absorvedora para reduzir as perdas por condução, conforme a figura 7 demonstra.

Figura 7: Detalhamento da placa coletora utilizada na bancada.

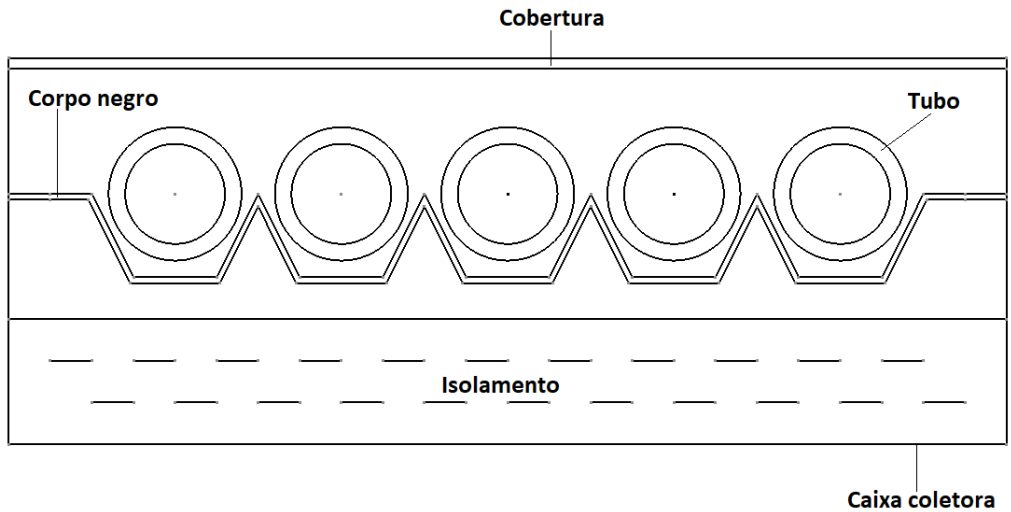

Fonte: Próprio autor.

\subsection{Trilha Do Conhecimento}

O modelo de estudo proposto seguirá uma linha de raciocínio pré-definida, denominada como "Trilha do Conhecimento", um material formatado no qual toda a razão logica de análise é apresentada em etapas de estudo. A "Trilha do Conhecimento" descrita neste estudo foi baseada no modelo teórico desenvolvido por (Duffie e Beckman, 2013) junto à bancada do coletor solar de placa plana (CPP) presente na área de testes da UERJResende, a "Trilha do Conhecimento" seguirá o roteiro abaixo da figura 8 para o desenvolvimento do trabalho.

Figura 8:Tabela Trilha do conhecimento

\begin{tabular}{|l|l|}
\hline Etapa 1 & Apresentacão ao conteúdo \\
\hline Etapa 2 & Aquisicão de dados do coletor de placa plana \\
\hline Etapa 3 & Consultar tabela de propriedades do ar \\
\hline Etapa 4 & Consultar dados do Instituto Nacional de Meteorologia (INMET) \\
\hline Etapa 5 & Aplicar os valores na planilha de dados \\
\hline Etapa 6 & Analisar os resultados obtidos \\
\hline
\end{tabular}

Fonte: Próprio Autor

Inicialmente os alunos são apresentados ao conteúdo através de palestra sobre os tipos de coletores solares presentes atualmente no mercado brasileiro, fazendo uma diferenciação dos modelos que, além do coletor de placa plana (CPP), temos também o coletor estacionário parabólico composto (CPC) e o coletor de tubos evacuados (CTE).

A aquisição de dados da bancada é realizada através de um equipamento de aquisição modelo Lynx ADS0500. Os termopares utilizados são do modelo tipo $\mathrm{K}$ e são devidamente calibrados antes da instalação no equipamento. É sugerido ao aluno a 
formatação de uma tabela de controle, a ser preenchida com os valores aquisitados durante a execução do experimento, conforme mostrado na figura 9.

Figura 9: Modelo proposto para monitoramento térmico da placa coletora.

\begin{tabular}{|c|c|c|c|c|c|c|c|c|c|c|c|c|c|c|c|c|c|c|c|c|c|}
\hline Dodis: & $\frac{1}{08000}$ & & $\frac{2}{08110}$ & & 0820 & rrigida & $\mid$ & Corrigida & \begin{tabular}{|l|l}
08440 \\
0
\end{tabular} & rrigida & $\frac{6}{0850}$ & rigida f & 09.00 & arig & \begin{tabular}{|c|}
8 \\
0910
\end{tabular} & Corrigida & $\frac{9}{00200}$ & jida & \begin{tabular}{|c|}
10 \\
0930
\end{tabular} & Corrigida & Medias \\
\hline benter & 27,53 & 26,913622 & 28,13 & 5020262 & 28,00 & 27,3967 & 27,15 & 26.52344 & 27,96 & 3555604 & 27,53 & 26,913822 & $27,53]$ & 69,913222 & 2753 & 8913822 & 1,20 & 269,93822 & $227,53]$ & 69.913822 & 7,028 \\
\hline Antes Do Bolief & 24,35 & 2379936 & 25,65 & 25,1233285 & 25,42 & 24,888118 & 2463 & 4,080027 & 24,34 & 237783386 & 24,35 & 23,798615 & 24,35 & 23,793615 & 24,35 & 7993615 & 24,35 & 23799615 & 24,35 & 779361! & 24,0308 \\
\hline Entrado Da Place & 35,41 & 35,918273 & 35,75 & 36,2686875 & 36,03 & 36,553959 & 35,88 & 36,600164 & 36,21 & 367,738513 & 35,41 & 35998273 & 35,41 & 35,98273 & 35,41 & 59,98273 & 35,41 & 35,918273 & 35,41 & 918273 & 36,146 \\
\hline 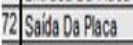 & 45,98 & 46,963238 & 46,91 & 47,878821 & 46,99 & 47,448669 & 45,96 & 46,895876 & 46,85 & 47,806435 & 45,98 & 46,96038 & 45,98 & 46,916338 & \begin{tabular}{|l|l|}
45,98 \\
\end{tabular} & 4,916338 & 45,98 & 46,96338 & 45,98 & 46,96338 & 47,2017829 \\
\hline Consumo & 55,82 & 55,63142 & 55,17 & 54,9605994 & 55,91 & 55,723062 & 55,66 & 55,468512 & 56,15 & 55,96743 & 55,82 & 556,631424 & 55,82 & 556,631424 & 55,82 & 56,631424 & 55,82 & 556,631424 & 55,82 & 556,631424 & 55,5917 \\
\hline Sidro Suverior & 84,32 & 84,692316 & 85,01 & 853997013 & 85,47 & 858868811 & 84,12 & 84,480565 & 84,01 & 84,375713 & 84,32 & 84,692316 & 84,32 & 84692316 & 84,32 & 84,62316 & 84,32 & 84,692316 & 84,32 & 84692316 & 84,2821489 \\
\hline Vidro heteror & 84,96 & 80,399788 & 85,42 & 88.80276 & 85.98 & 87,433044 & 8432 & 85,735196 & 8427 & 85684056 & 84,96 & 80.389788 & 84.96 & 86,389788 & 84,96 & 80,399788 & 84,96 & 863899788 & 8496 & 863399788 & 86,4513 \\
\hline 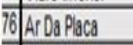 & 94,08 & 95,717724 & 95,62 & 97,292836 & 97,66 & 99,379348 & 94,19 & 95,802322 & 95,78 & 97,456484 & 94,08 & 95,717724 & 94,08 & 95,717724 & 94,08 & 055,71724 & 94,08 & 95,717724 & 94,08 & 95,717724 & 96,2255244 \\
\hline 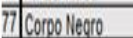 & & & & & & & & & & & & & & & & & & & & & \\
\hline
\end{tabular}

Fonte: Próprio Autor

Seguindo a "Trilha do Conhecimento", os alunos passarão para a parte teórica, onde usarão as ferramentas necessárias para os cálculos propostos da análise do coletor. Nem todas as variáveis serão provenientes da aquisição experimental, desta forma os alunos consultarão uma tabela de propriedades do ar, na literatura e irão formatar uma tabela de análise em função da temperatura $(T)$, interpolando para a utilização dos dados como viscosidade dinâmica $(\eta)$, massa especifica $(\rho)$, condutividade térmica do material $(K)$ e o calor especifico $(C p)$, deste modo determinando as variáveis essenciais para os cálculos do modelo proposto.

Figura 10: Tabela utilizada para determinar as variáveis.

\begin{tabular}{|c|c|c|c|c|}
\hline $\boldsymbol{T}$ & $\boldsymbol{\eta}$ & $\boldsymbol{\rho}$ & $\boldsymbol{K}$ & $\boldsymbol{C p}$ \\
\hline${ }^{\circ} \mathrm{C}$ & $\mathrm{Cp}$ & $\mathrm{kg} / \mathrm{m}^{3}$ & $\mathrm{kcal} / \mathrm{m} \mathrm{h}^{\circ} \mathrm{C}$ & $\mathrm{kcal} / \mathrm{kg}^{\circ} \mathrm{C}$ \\
\hline 0 & 0,01729 & 1,292 & 0,02033 & 0,24028 \\
\hline 5 & 0,01754 & 1,269 & 0,02064 & 0,24028 \\
\hline 10 & 0,01778 & 1,246 & 0,02097 & 0,24028 \\
\hline 15 & 0,01802 & 1,225 & 0,02129 & 0,24052 \\
\hline 20 & 0,01825 & 1,204 & 0,02162 & 0,24052 \\
\hline 25 & 0,01849 & 1,184 & 0,02193 & 0,24052 \\
\hline 30 & 0,01872 & 1,164 & 0,02225 & 0,24052 \\
\hline 35 & 0,01895 & 1,145 & 0,02257 & 0,24052 \\
\hline 40 & 0,01918 & 1,127 & 0,02289 & 0,24052 \\
\hline 45 & 0,01941 & 1,109 & 0,02321 & 0,24052 \\
\hline 50 & 0,01963 & 1,092 & 0,02352 & 0,24052 \\
\hline
\end{tabular}

Fonte: Adaptado de Incropera (2008)

Outro fator determinante para os cálculos é a velocidade do vento na região, pelo fato de não haver o equipamento necessário para fazer essa medição, serão consultados os dados do Instituto Nacional de Meteorologia (INMET), com informações da estação automático de Resende (A609), no qual a velocidade do vento será considerada o valor médio dos valores obtidos nas horas em que aquisição dos dados fora realizada, conforme a tabela da figura 11 mostrada abaixo. 
Figura 11: Dados de monitoramento do INMET.

\begin{tabular}{|c|c|c|c|c|c|c|c|c|c|c|c|c|c|c|c|c|c|c|}
\hline \multirow{2}{*}{ Data } & \multirow{2}{*}{$\begin{array}{l}\text { Hora } \\
\text { UTC }\end{array}$} & \multicolumn{3}{|c|}{ Temperatura $\left({ }^{\circ} \mathrm{C}\right)$} & \multicolumn{3}{|c|}{ Umidade (\%) } & \multicolumn{3}{|c|}{ Ponto De Orvalho $\left({ }^{\circ} \mathrm{C}\right)$} & \multicolumn{3}{|c|}{ Pressão (hPa) } & \multicolumn{3}{|c|}{ Vento } & \multirow{2}{*}{$\begin{array}{c}\text { Radiação } \\
\mathrm{Kj} / \mathrm{m}^{2}\end{array}$} & \multirow{2}{*}{$\begin{array}{l}\text { Chuva } \\
\text { (mm) }\end{array}$} \\
\hline & & Inst. & Máx. & Min. & Inst. & Máx. & Min. & Inst. & Máx. & Min. & Inst. & Máx. & Min. & Vel. $(\mathrm{m} / \mathrm{s})$ & Dir. $\left({ }^{\circ}\right)$ & Raj.(m/s) & & \\
\hline 03/03/2021 & 8 & 18,600 & 19,600 & 18,600 & 92,000 & 92,000 & 90,000 & 17,300 & 18,300 & 17,200 & 965,000 & 965,000 & 964,700 & 0,700 & 276,000 & 2,100 & 62,980 & 0,000 \\
\hline 03/03/2021 & 9 & 18,400 & 19,200 & 18,200 & 94,000 & 94,000 & 92,000 & 17,400 & 18,200 & 16,900 & 965,400 & 965,400 & 965,000 & 0,600 & 204,000 & 1,600 & 105,170 & 0,000 \\
\hline Média & & 18,500 & 19,400 & 18,400 & 93,000 & 93,000 & 91,000 & 17,350 & 18,250 & 17,050 & 965,200 & 965,200 & 964,850 & 0,650 & 240,000 & 1,850 & 84,075 & 0,000 \\
\hline
\end{tabular}

Fonte: Próprio autor

Em continuidade desta proposta pedagógica, os alunos terão acesso a um banco de dados adequado a análise térmica do coletor, o que permite a interação a todo equacionamento necessário para o desenvolvimento da técnica teórico-experimental, permitindo o discente de explorar todas as etapas do projeto e desenvolvimento. Como observado na figura 12, o roteiro funcionará como um grande facilitador do entendimento, seguindo o passo-a-passo para buscar os resultados desejados.

Figura 12: Planilha orientativa.

\begin{tabular}{|c|c|c|c|}
\hline Simbologia & Dados & Valor & Unidade \\
\hline$T_{a}$ & Temperatura ambiente do ar & 300,17889 & $\mathrm{~K}$ \\
\hline$T_{s}$ & Temperatura do céu & 300,1788908 & $\mathrm{~K}$ \\
\hline$T_{0}$ & Temperatura da cobertura & 359,55513 & $\mathrm{~K}$ \\
\hline$T_{p}$ & Temperatura da placa & 385,4511475 & $\mathrm{~K}$ \\
\hline$T_{p m}$ & Temperatura média da placa & 385,4511475 & $\mathrm{~K}$ \\
\hline$T_{\mathrm{ar}}$ & temperatura do ar dentro da placa & 369,5765244 & $\mathrm{~K}$ \\
\hline$\Delta T$ & Diferença de temperatura entre as placas & 25,89602 & $\mathrm{~K}$ \\
\hline$t$ & Temperatura média & 372,5031388 & $\mathrm{~K}$ \\
\hline$v$ & Velocidade do ar & 1,150 & $\mathrm{~m} / \mathrm{s}$ \\
\hline 1 & Largura do coletor & 0,876 & $\mathrm{~m}$ \\
\hline$c$ & Comprimento do coletor & 1,03 & $\mathrm{~m}$ \\
\hline$a$ & Altura do coletor & 0,0541 & $\mathrm{~m}$ \\
\hline$e$ & Espessura do vidro & 0,00325 & $\mathrm{~m}$ \\
\hline$L$ & Distância entre a cobertura e a placa absorvedora & 0,03 & $\mathrm{~m}$ \\
\hline$P_{c}$ & Perimetro do coletor & 3,812 & $\mathrm{~m}$ \\
\hline$A_{c}$ & Área do coletor & 0,90228 & $\mathrm{~m}^{2}$ \\
\hline$V_{c}$ & Volume do coletor & 0,048813348 & $\mathrm{~m}^{\mathrm{s}}$ \\
\hline$\beta$ & ângulo de inclinação do coletor & 22,46 & $\circ$ \\
\hline$g$ & Aceleração da gravidade & 9,807 & $\mathrm{~m} / \mathrm{s}^{2}$ \\
\hline$\eta$ & Viscosidade Dinâmica & 0,00001860 & $\mathrm{~kg} / \mathrm{ms}$ \\
\hline$p$ & Densidade & 1,164 & $\mathrm{~kg} / \mathrm{m}^{\mathrm{s}}$ \\
\hline$K$ & Condutividade térmica & 0,0264001 & $\mathrm{~W} / \mathrm{m}^{2} \mathrm{~K}$ \\
\hline$C p$ & Calor especifico & 1004,832 & $\mathrm{~J} / \mathrm{kg} . \mathrm{K}$ \\
\hline $\bar{\sigma}$ & Constante de Stefan-Boltzman & $5,67 \mathrm{E}-08$ & $W /\left(m^{2} K^{4}\right)$ \\
\hline$\varepsilon_{p}$ & Emissividade da placa & 0,95 & \\
\hline$\varepsilon_{0}$ & Emissividade da cobertura & 0,88 & \\
\hline $\mathrm{K}_{\mathrm{iso}}$ & Condutividade térmica do material isolante & 0,046 & $\mathrm{~W} / \mathrm{m} \cdot \mathrm{K}$ \\
\hline$L_{\text {iso }}$ & Espessura do material isolante & 0,020 & $\mathrm{~m}$ \\
\hline
\end{tabular}

Fonte: Próprio autor

Os dados mostrados na figura 12, refletem a entrada de informações no qual o aluno é instigado a entender as condições do pré-dimensionamento do coletor solar. Com todas as variáveis já declaradas no processo anterior, o banco de dados conforme mostrado na figura 13, gerará os resultados do projeto, neste momento os alunos serão orientados a analisar as informações e promover a discussão em grupo, aplicando os conhecimentos adquiridos ao longo de sua formação acadêmica, baseando-se na análise térmica, serão 
conduzidos neste momento pelo professor como mediador e facilitador que auxiliará na discussão e na análise de dados dos resultados obtidos.

Figura 13: Tabela proposta para os resultados obtidos

\begin{tabular}{|c|c|c|c|}
\hline Simbologia & Dados & Valor & Unidade \\
\hline$v$ & Viscosidade cinemática & $1,60825 \mathrm{E}-05$ & $\mathrm{~m}^{2} / \mathrm{s}$ \\
\hline$a$ & Difusidade térmica & $2,21268 \mathrm{E}-05$ & $\mathrm{~m}^{2} / \mathrm{s}$ \\
\hline Pr & Número de Prandtl & 0,726833804 & - \\
\hline$R a$ & Número de Rayleigh & 51728,79622 & - \\
\hline $\mathrm{Nu}$ & Número de Nusselt & 1,025219580 & - \\
\hline$H_{w}$ & Coeficiente de transferência de calor por convecção do ar & 4,200573665 & $\mathrm{~W} / \mathrm{m}^{20} \mathrm{C}$ \\
\hline$H_{r, p-0}$ & Coeficiente de transferência de calor por radiação entre a placa e cobertura & 9,871363839 & $\mathrm{~W} / \mathrm{m}^{20} \mathrm{C}$ \\
\hline$H_{r, 0-a}$ & Coeficiente de transferência de calor por radiação entre a cobertura e o ambiente & 7,221809219 & $\mathrm{~W} / \mathrm{m}^{20} \mathrm{C}$ \\
\hline$H_{0, p-0}$ & Coeficiente de transferência de calor por convecção entre a placa e a cobertura & 0,884422579 & $\mathrm{~W} / \mathrm{m}^{20} \mathrm{C}$ \\
\hline$R 1$ & Resistência 1 & 0,087547407 & $\mathrm{~W} / \mathrm{m}^{20} \mathrm{C}$ \\
\hline$R 3$ & Resistência 3 & 0,092973211 & $\mathrm{~W} / \mathrm{m}^{20} \mathrm{C}$ \\
\hline$U_{t}$ & Coeficiente global do topo & 5,539533449 & $\mathrm{~W} / \mathrm{m}^{20} \mathrm{C}$ \\
\hline$U_{b}$ & Coeficiente global da base & 2,300000000 & $\mathrm{~W} / \mathrm{m}^{20} \mathrm{C}$ \\
\hline$U_{e}$ & Coeficiente global da borda & 0,525698408 & $\mathrm{~W} / \mathrm{m}^{20} \mathrm{C}$ \\
\hline$U_{L}$ & Coeficiente global total & 8,365231857 & $\mathrm{~W} / \mathrm{m}^{20} \mathrm{C}$ \\
\hline
\end{tabular}

Fonte: Próprio Autor

\subsection{Aplicação Extensionista}

O Laboratório de Fenômenos de Transporte, está diretamente envolvido com atividades extensionistas, seja no acompanhamento de visitas de escolas e instituições, como no apoio ao projeto "UERJ no Campo". Os alunos do curso de Fenômenos de Transporte Experimental e os alunos voluntários do Laboratório de Fenômenos de Transporte, são estimulados a participar destas atividades, através do acompanhamento de visitas, realização de palestras, oficinas e outras atividades. Recentemente, com 0 desenvolvimento da proposta do projeto extensionista "Coletor Solar Itinerante", figura 12 e figura 13, uma proposta de parceria entre UERJ e o Sindicato Rural de Resende, onde professores e alunos, projetaram e desenvolveram um conjunto coletor solar desmontável, com objetivos de visitar as propriedades rurais sul fluminense, ainda torna possível desenvolver o esclarecimento dos benefícios do uso da energia renovável, assim como detalhes de custo e benefício ao produtor rural. 
Figura 14: Desenho do projeto.

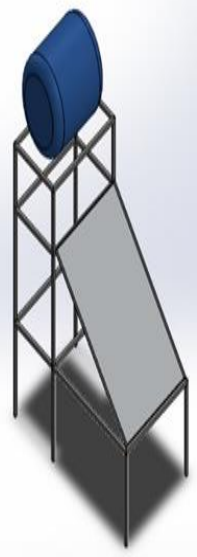

Fonte: Próprio Autor
Figura 15: Protótipo montado na área de testes.

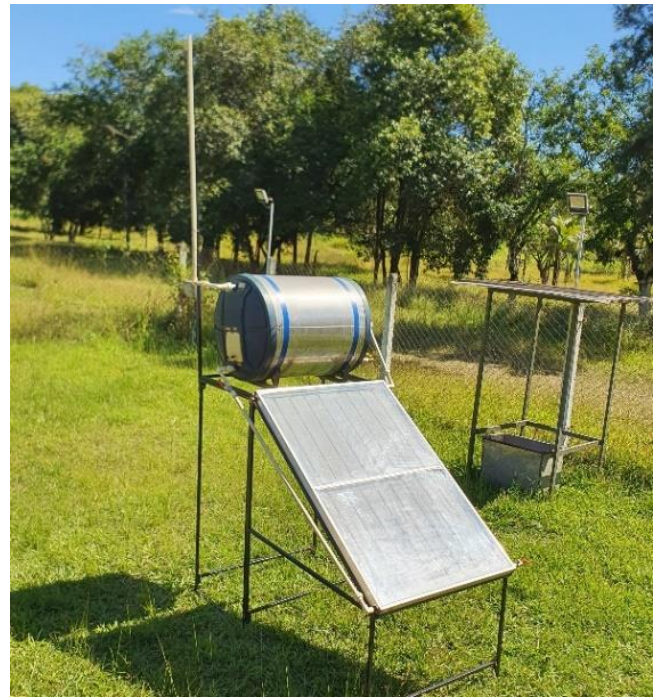

Fonte: Próprio Autor

Além de todos as atividades anteriormente descritas no desenvolvimento técnico dos alunos de engenharia, através de metodologias ativas e a proposta da "Trilha do Conhecimento", os mesmos ainda são estimulados a desenvolver suas competências socioemocionais, frente as ações junto à comunidade rural $e$ as dificuldades compartilhadas. Desta forma, são estimulados a se desenvolver através da empatia e das habilidades que serão motivadas frente a realização de palestras, oficinas e demais atividades.

Figura 16: Competências socioemocionais.

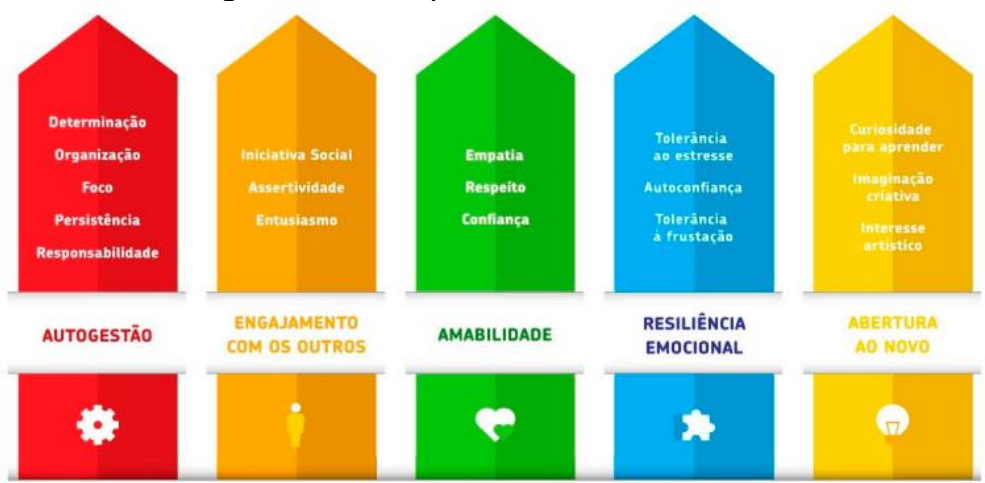

Fonte: InstitutoAytonSenna.org.br

\section{AVALIAÇÃO DOS RESULTADOS}

Ao desenvolver a proposta de utilizar o experimento do Coletor Solar nas atividades da disciplina de Fenômenos de Transporte Experimental, a proposta foi de conciliar a base teórica já estudada pelos discentes nas disciplinas de Transferência de Calor e Mecânica dos Fluidos, nas atividades práticas, onde os alunos são motivados a desenvolver as habilidades nas análises e nas percepções dos eventos físicos específicos ao projeto.

Implementar a "Trilha do Conhecimento", foi uma ação muito positiva, uma vez que disciplina os alunos na organização das ideias, na condução do raciocínio lógico e no procedimento de utilização de ferramentas de medição e interpretação dos resultados. 
A possibilidade de extrapolar a fronteira da sala de aula, através de ações Extensionistas, permite ao aluno um outro olhar na questão estudada, através do desenvolvimento da empatia, o aluno desenvolve além desta técnica, outras habilidades diversificando o estudo em fenômenos de transporte usualmente praticado.

O ganho de maturidade e autoconfiança, frente as questões extensionista, refletem em atividades secundárias, como ideias empreendedoras através de propostas em Trabalhos em Conclusão de Curso em "Desidratadores de Alimentos" e ou "Sistemas Purificadores de Água", entre outros, visto que o problema é problema quando não se tem a técnica e ou o preparo necessário, e em outros olhos é visto como oportunidades.

\section{CONCLUSÃO}

A partir do trabalho realizado, pode-se concluir que a atividade proposta cumpre o objetivo de melhorar 0 aprendizado, estimular 0 desenvolvimento de diversas competências, assim como o empreendedorismo e principalmente a valorização da utilização dos recursos das energias renováveis. Conciliar teoria e prática, através de ferramentas pedagógicas e extensionistas transforma o ensino experimental em um processo mais dinâmico, e entrega ao aluno mais motivação e autoconfiança no futuro profissional.

\section{REFERÊCIAS BIBLIOGRÁFICAS}

CARVALHO, Filipe R., SIONEK, Mirella C. ANÁLISE DA EFICIÊNCIA DE UM COLETOR SOLAR PLANO. 2015. Monografia (Graduação) - Curso de Engenharia Mecânica, Universidade Tecnológica Federal do Paraná, Curitiba, 2015. Disponível: http://repositorio.roca.utfpr.edu.br/jspui/bitstream/1/6597/1/CT_COEME_2014-2_05.pdf.

Acesso em: 15 mar. 2021

DUFFIE, John A., BECKMAN, William A. Solar engineering of thermal processes. 4 ed, New York: Wiley, 2013.

INCROPERA, Frank P. et al. Fundamentos de Transferência de Calor e de Massa. 6 ed, Rio de Janeiro: LTC, 2008.

PEREIRA, Enio B. et al. Atlas brasileiro de energia solar. 1. ed, São José dos Campos: INPE, 2006.

ABSTRACT: In the proposed article, the theoretical and experimental development of a didactic bench for the thermal efficiency analysis of a flat plate solar collector is presented, in addition to the formation of the discipline of Experimental Transport Phenomena of the Mechanical Engineering course at the State University Rio de Janeiro, Resende unit. Initially, students are encouraged to develop the analytical model of the solar collector, reconciling all academic training in the disciplines of heat transfer and fluid mechanics. In the development of the experiment, data acquisition techniques are inserted, through the use of various means of measuring temperature and flow, as well as consulting official environmental monitoring data. In parallel, volunteer students are encouraged to participate in extension activities, "Projeto UERJ no Campo" in which they seek to meet the needs of rural communities, through this renewable resource, developing in these students' skills and 
stimulating entrepreneurial actions in the search for solutions that can meet to this community reconciling cost and benefit. The extension project also extends to other duties, as a means of spreading information, knowledge, inclusion and accessibility, encouraging students to give lectures and workshops to both the rural community and public and private schools in the South of Rio de Janeiro.

Keywords: Experimental Transport Phenomena; Flat Plate Solar Collector; Extension Activities 